\title{
Cárie dentária e condições sócio-econômicas no Estado do Paraná, Brasil, 1996
}

\author{
Dental caries and socioeconomic conditions \\ in the State of Paraná, Brazil, 1996
}

Márcia Helena Baldani 1

Paulo Capel Narvai 2

José Leopol do Ferreira Antunes 3

\footnotetext{
1 Faculdade de Odontol ogia, Universidade Estadual de Ponta Grossa. Av. Carlos Cavalcante 4748, Bloco M, Campus de Uvaranas, Ponta Grossa, PR 84030-000, Brasil. marcia@convoy.com.br 2 Faculdade de Saúde Pública, Universidade de São Paulo.

Av. Dr. Arnaldo 715 São Paulo, SP 01246-904, Brasil. pcnarvai@usp.br

3 Faculdade de Odontologia, Universidade de São Paulo. Av. Prof. Lineu Prestes 2227, São Paulo, SP 05508-900, Brasil. leopoldo@fo.usp.br
}

\begin{abstract}
The aim of this research was to determine the correlation between dental caries and socioeconomic conditions in the State of Paraná, Brazil. Caries prevalence was estimated for each city in the State by gathering data on the DMFT index (in 12-year-old schoolchildren) supplied by the State Health Department. Official socioeconomic data for the municipaliti es were also presented. Ordinary least squares regression analysis was performed, and si gnificant correlation coefficients were observed between the dental caries index and various social development indicators. Maps highlighting the overlapping areas with poor outcomes for most of these variables were presented. The results showed a significantly lower DMFT index in cities with fluoridated water supply and a negative correlation between the caries index and the proportion of households with running water in cities with fluoridated water supply. This observation highlights the importance of fluoridated drinking water for the prevention of dental caries and as a measure to reduce the impact of socioeconomic inequalities on the prevalence of tooth decay. Key words Dental Caries; Social Conditions; Oral Health; Prevalence; Epidemiology
\end{abstract}

Resumo O presente estudo foi conduzido com o objetivo de anal isar as relações entre cárie dentária e fatores sócio-econômicos no Estado do Paraná, Brasil. Foram reuni das informações sobre a prevalência de cárie dentária (CPO-D aos 12 anos) para os municípios do Estado, conforme dados oficiais disponi bilizados pel a Secretaria de Estado da Saúde. Pôde-se observar correlação si gni fi cante entre o índice de cárie dentária nos municí pios e vários indi cadores de desenvol vimento social, através da análise de regressão linear simples. A rel ação visual entre as ci dades com piores figuras de CPO-D e indicadores sociais, foi apresentada em mapas através do georreferenciamento de dados. Observou-se CPO-D médi o menos el evado nos municí pios cujos reservatórios de água foram fluorados. Observou-se também, correl ação negativa entre o índice de cárie dentária e a proporção de domicílios ligados à rede de abasteci mento de água, nos municípios com água fluorada. Nesse sentido, subl inha-sea importância desse benefício, não só como recurso para a redução dos níveis de cáries, como também para atenuar o impacto das desigualdades sócio-econômicas sobre a prevalência de cárie dentária.

Palavras-chave Cárie Dentária; Condi ções Sociais; Saúde Bucal; Prevalência; Epi demi ologia 


\section{Introdução}

O Ministério da Saúde (MS), divulgou informações sobre a prevalência de cárie dentária no país, obtidas em pesquisa realizada em 1996 (MS, 2001). Segundo essas informações, o CPOD aos 12 anos de idade, registrou média de 2,23 para o Paraná. Como a investigação teve por base a situação observada apenas nas capitais estaduais, deu margem a várias interrogações: seriam estes valores representativos para os demais municípios? Haveria homogeneidade na distribuição da doença no Estado? Como a cárie dentária se relacionaria com diferentes aspectos sócio-econômicos, reconhecidamente heterogêneos?

A epidemiologia é um poderoso instrumento no campo do planejamento em saúde. Assim, obter respostas para essas questões é uma importante tarefa para os profissionais da área, uma vez que a definição de programas de prevenção e tratamento das doenças bucais, bem como o planejamento dos serviços, devem ser instruídos pelo resultado de estudos epidemiológicos. Quando criteriosos, realizados periodicamente em nível local, regional ou nacional, e em condições homogêneas, esses estudos permitem identificar, avaliar e monitorar a distribuição e tendências da prevalência e severidade das doenças ( $M$ arcenes \& Bonecker, 2000). Os levantamentos básicos devem produzir dados confiáveis, possibilitando assim o desenvolvimento de bons programas de saúde bucal e o planejamento adequado da quantidade e do tipo de profissionais necessários para a sua execução.

Observa-se, porém, que as informações epidemiológicas obtidas em nível municipal, são pouco utilizadas e, muitas vezes, sequer chegam a ser publicadas. Com isso, esses estudos são sub-utilizados e seus achados e conclusões poucas vezes derivam conseqüências efetivas, ficando inexplorado o potencial dos dados produzidos (Vaughan \& Morrow, 1997).

Em 1986, o MS havia realizado um levantamento epidemiológico de saúde bucal em nível nacional, no qual foram coletados dados referentes à cárie dentária, doença periodontal, necessidades de prótese e acesso aos serviços odontológicos da população urbana de 16 capitais (MS, 1988). Apesar das críticas já apontadas ao levantamento realizado em 1996, a análise comparativa dos resultados de ambos os estudos apontou uma queda de $54,1 \%$ no índice CPO-D nacional para 12 anos de idade: de 6,67 em 1986, o índice passou a 3,06 em 1996 (Narvai et al., 1999).

Apesar de alguns autores admitirem que as condições de saúde bucal melhoraram nas úl- timas décadas (Nadanovsky, 2000; Pinto, 1996 1997; Weyne, 1997), a cárie dentária permanece como um grande problema de saúde pública, tanto no Brasil (Andrade, 2000; MS, 1988, 2001) como na maior parte do mundo (Loretto et al., 2000; Martins et al., 1999; Weyne, 1997). Como possíveis causas para o declínio observado nos índices de cárie, os autores citam a adição de flúor à água de abastecimento público, o emprego em larga escala de dentifrícios fluorados e a reforma dos serviços de saúde, que acompanharam a implantação do Sistema Único de Saúde. Outras causas importantes seriam, em alguns países, o consumo diferenciado de açúcares e a melhoria nas condições de vida da população (Marcenes \& Bonecker, 2000; Nadanovsky, 2000; Pinto, 1997, 2000; Weyne, 1997).

No entanto, esses mesmos estudos apontam que a melhoria do nível global dos indicadores de cárie dentária, foi acompanhada pela polarização do agravo nos grupos de população mais submetidos à privação social. O quadro epidemiológico brasileiro, em geral, e do Estado do Paraná, em particular, expressa a persistência de importantes desigualdades sócioculturais, econômicas e políticas. A condição social tem sido enfatizada nas últimas décadas, como importante determinante das condições de saúde bucal (Furlani, 1993; Loretto et al., 2000; Martins et al., 1999; Nadanovsky, 2000). Vários trabalhos têm abordado a correlação entre classe social e cárie dentária ou doença periodontal (Jones \& Worthington, 2000; Marcenes \& Bonecker, 2000; Peres et al ., 2000). Têmse observado grupos específicos de população que permanecem com elevada prevalência de cárie dentária; de modo geral, a especial vulnerabilidade ao agravo está associada à exposição mais intensa aos fatores de risco e à privação social (Martins et al., 1999). Em alguns estudos, observou-se que a prevalência de cárie diminuiu na medida em que o nível sócio-econômico aumentou, mesmo em áreas sem a adição de flúor à água de abastecimento público (Jones \& Worthington, 2000; Marcenes \& Bonecker, 2000).

Ao analisar os fatores sócio-econômicos relacionados com a cárie dentária, deve-se considerar a etiologia das desigualdades sociais, como a má distribuição da renda, a falta de participação na riqueza nacional, o desemprego, o atraso tecnológico em alguns setores e os elevados índices de analfabetismo. Além das dificuldades de acesso aos serviços odontológicos, pessoas com diferenças pronunciadas de renda também estão em desvantagem quanto à ocorrência de problemas de saúde bucal. Tal constatação foi registrada no levantamento do 
MS em 1986, quando se indicou que as pessoas situadas nos estratos de renda mais elevada, possuem menos cáries do que as situadas na base da pirâmide sócio-econômica (Pinto, 1997).

Com base nessas observações, propôs-se abordar, no presente estudo, as relações entre cárie dentária e fatores sócio-econômicos nos municípios do Estado do Paraná, Brasil, em 1996. O objetivo foi identificar as características da prevalência de cárie dentária nos municípios do Estado, e determinar a correlação entre esses índices e indicadores de desenvolvimento social.

\section{Material e métodos}

Os dados foram obtidos através da revisão de documentos e consultas aos bancos de dados do MS e do IBGE (Fundação Instituto Brasileiro de Geografia e Estatística). Os indicadores de prevalência de cárie foram fornecidos por levantamento epidemiológico em saúde bucal, realizado em 1996, sob a responsabilidade técnico-científica da Secretaria de Estado da Saúde do Paraná (SES-PR, 1996a, 1996b), e abrangeram 357 dos 371 municípios do Estado, tendo sido o estudo especificamente dirigido para estimar o índice CPO-D em escolares de 12 anos de idade. A metodologia empregada para o levantamento, seguiu as diretrizes internacionais estipuladas pela OMS (Organização Mundial da Saúde) em 1997. O relatório desse levantamento afirma ter sido realizada a calibração dos examinadores, mas não fornece informações sobre indicadores de concordância intra e interexaminadores. Nesse sentido, o presente artigo admitiu a viabilidade da análise dos dados obtidos naquele estudo transversal.

O índice CPO-D é um instrumento largamente utilizado em pesquisas epidemiológicas de cárie dentária sendo, por esta razão, recomendado pela OMS para medir e comparar a prevalência de cárie dentária em populações. Seu valor expressa a média do número de dentes permanentes cariados, extraídos "perdidos" e restaurados "obturados" num grupo de indivíduos (OMS, 1999). A idade de 12 anos, foi utilizada por ser uma das idades-índice preconizadas pela OMS para estudos de saúde bucal em populações (Narvai et al., 2000).

Os aspectos sociais foram evidenciados através de diferentes indicadores de desenvolvimento, envolvendo condições de saúde, renda, moradia e educação. Foram escolhidos para a análise, somente aqueles indicadores cujos dados estavam disponíveis para todos os municípios que realizaram o levantamento de cárie dentária. Como índices globais, foram incluídos o Índice de Desenvolvimento Humano (IDH), o Índice de Condições de Vida (ICV), o Índice de Salubridade (IS) e o Índice de Desenvolvimento Infantil (IDI). Além destes, foram incluídos outros indicadores: (a) relativos à renda: renda familiar média per capita (medida em número de salários mínimos), índice de Theil (indicador da desigual dade na distribuição de renda) e renda insuficiente (porcentagem de população vivendo com renda média familiar per capita inferior a meio salário mínimo); (b) relaci onados com a moradia: agl omeração domiciliar (porcentagem de população vivendo em domicílios com mais de dois moradores por dormitório), proporção de domicílios construídos com materiais duráveis, domicílios com ligação adequada às redes de água e esgoto e (c) relati vos à escolaridade: coeficiente de analfabetismo em adultos ( 25 anos ou mais), anos médios de estudo, proporção de crianças (de 7 a 14 anos) sem escola, defasagem escolar média, crianças (de 7 e 14 anos) com mais de um ano de atraso na escola e proporção de crianças (de 7 e 14 anos) que trabalham.

$\mathrm{O}$ IDH foi elaborado pelo Programa das $\mathrm{Na}-$ ções Unidas para o Desenvolvimento (PNUD) e é largamente utilizado em estudos sobre condições de vida. Este indicador parte da concepção de que renda, saúde e educação são três elementos fundamentais da qualidade de vida de uma população. A renda é avaliada por indicadores médios e pela desigual dade da distribuição; a saúde, pela esperança de vida ao nascer e mortalidade infantil e a educação, pela taxa de alfabetização de adultos e taxas de matrículas nos níveis primário, secundário e terciário combinados (Minayo et al., 2000).

O ICV foi originalmente desenvolvido pela Fundação João Pinheiro, de Belo Horizonte, para estudar a situação de municípios mineiros e, posteriormente, foi modificado e adequado para a análise de todos os municípios brasileiros. É composto de vinte indicadores em cinco dimensões: renda, educação, infância, habitação e longevidade (M inayo et al., 2000).

O IS foi desenvolvido na Secretaria de Estado da Saúde do Paraná, com o objetivo de subsidiar o processo de implantação da estratégia de “municípios saudáveis”. É um indicador sintético, que permite classificar os municípios segundo suas posições em um ranking composto por 29 indicadores sociais, de saúde e de serviços, organizados nas categorias de mortalidade, morbidade, sócio-ambientais e oferta de serviços (Strozzi, 1997).

O IDI foi desenvolvido pelo Fundo das $\mathrm{Na}$ ções Unidas para a Infância (UNICEF), tendo 
sido calculado no Brasil em nível municipal. Foi composto a partir de indicadores relacionados a dimensões do desenvolvimento infantil, como cobertura vacinal contra sarampo, grau de escolaridade dos pais, renda familiar, acesso à água tratada, entre outros (UNICEF, 2001).

Os indicadores referentes à renda, domicílio e grau de instrução são relativos ao Censo de 1991 (IBGE, 1994).

A análise estatística foi efetuada através do SPSS 8.0 (SPSS Incorporation, 1997). O estudo da correlação entre cárie dentária e os diversos indicadores sociais, foi efetuado através de análise de regressão linear simples, conforme descrito por Johnson \& Wichern (1998). O índice CPO-D aos 12 anos, bem como alguns dos indicadores, foram organizados em mapas com o uso de georreferenciamento. Para a disposição visual dos indicadores nos mapas, empregouse o programa Tabwin 1.4 (DATASUS, 2000).

\section{Resultados}

A Tabela 1, apresenta os valores médios do CPO-D para os municípios do Estado do Paraná, agrupados segundo a classificação de prevalência de cárie proposta pela OMS (Murray, 1992). Observa-se que a maioria dos municípios encontra-se nos grupos de alta $(45,94 \%)$ e moderada $(28,01 \%)$ prevalência de cárie. É também bastante expressivo o número de municípios que se apresentam na categoria muito alta $(18,77 \%)$. Quanto à média geral do CPO-D para o Estado, observou-se um valor de 5,15, valor considerado de alta prevalência, segundo os padrões da OMS.

A Tabela 2, mostra a distribuição dos municípios em relação às faixas de prevalência de cárie, indicando que a maioria das cidades de pequeno porte demográfico, isto é, com população de até 10.000 habitantes, apresentam-se na categoria de alta prevalência, seguindo-se a categoria de muito al ta prevalência. Também os municípios de médio porte, isto é, com população entre 10.000 e 50.000 habitantes, apresentam-se em maior número na categoria de alta prevalência de cárie, porém, já se observa um amplo contingente de municípios na categoria de moderada prevalência. A categoria de moderada prevalência de cárie, predomina entre os municípios de grande porte, isto é, com mais de 50.000 habitantes. Observa-se, ainda, uma inversão nos níveis de prevalência de cárie dentária mais freqüentes, entre os municípios de menor porte (com maior prevalência) e os de maior porte (com menor prevalência).

A Tabela 3, apresenta as correlações entre os indicadores sócio-econômicos utilizados e cárie dentária. Os níveis de cárie e os indicadores de desenvolvimento social (IDH, ICV, IS e IDI) apresentaram correlação negativa, indicando a tendência de municípios com melhores condições de vida apresentarem menores índices do agravo. A correlação entre os níveis de prevalência de cárie e as medidas de renda, indica a associação do agravo com a pobreza; a correlação com os indicadores de escolaridade, aponta a tendência de municípios com piores indicadores educacionais apresentarem também piores figuras de CPO-D. Também se observou, correlação negativa entre o CPO-D e os porcentuais de ligação à rede de abastecimento de água, indicando menores níveis do agravo nos municípios com maior oferta do serviço de águas. E, apesar de sua menor intensidade, a correlação com o indicador de aglomeração domiciliar, indica uma relação significante entre a distribuição de cáries e densidade de pessoas por domicílio.

Tabela 1

Índice CPO-D em escolares de 12 anos de idade, nos municípios do Estado do Paraná, segundo a classificação da Organização Mundial da Saúde dos níveis de prevalência de cárie dentária, em 1996.

\begin{tabular}{lcccc}
\hline Prevalência de Cárie & Média & Desvio Padrão & $\begin{array}{c}\text { Número de } \\
\text { Municípios }\end{array}$ & $\begin{array}{c}\text { Proporção de } \\
\text { Municípios }\end{array}$ \\
\hline Muito baixa & - & - & - & - \\
Baixa & 2,27 & 0,37 & 26 & 7,28 \\
Moderada & 3,64 & 0,55 & 100 & 28,01 \\
Alta & 5,38 & 0,65 & 67 & 45,94 \\
Muito alta & 7,96 & 1,54 & 357 & 18,77 \\
Total & 5,15 & 1,87 & 100,00 \\
\hline
\end{tabular}


Tabela 2

Distribuição dos municípios do Estado do Paraná, segundo a classificação da Organização Mundial da Saúde dos níveis de prevalência de cárie dentária em escolares de 12 anos de idade e o porte demográfico, em 1996.

\begin{tabular}{|c|c|c|c|c|c|c|c|}
\hline $\begin{array}{l}\text { Porte } \\
\text { Demográfico }\end{array}$ & baixa & $\begin{array}{l}\text { Prevalência } \\
\text { moderada }\end{array}$ & $\begin{array}{c}\text { de Cárie } \\
\text { alta }\end{array}$ & muito alta & $\begin{array}{l}\text { Número de } \\
\text { Municípios }\end{array}$ & CPO-D (média) & $\begin{array}{l}\text { Desvio } \\
\text { Padrão }\end{array}$ \\
\hline Pequenoa & 10 & 37 & 85 & 40 & 172 & 5,45 & 1,83 \\
\hline Médiob & 8 & 48 & 75 & 25 & 156 & 5,11 & 1,86 \\
\hline Grandec & 8 & 15 & 4 & 2 & 29 & 3,61 & 1,29 \\
\hline Total & 26 & 100 & 164 & 67 & 357 & 5,15 & 1,87 \\
\hline
\end{tabular}

a até 10.000 habitantes; b 10.000 a 50.000 habitantes; c mais de 50.000 habitantes.

Indicadores de desenvolvimento social, segundo a classificação da Organização Mundial da Saúde de níveis de prevalência de cárie dentária, coeficiente de correlação e nível de significância da correlação com o índice CPO-D em escolares de 12 anos de idade. Municípios do Estado do Paraná, 1996.

\begin{tabular}{|c|c|c|c|c|c|c|}
\hline Indicadores & Baixa & Moderada & Alta & Muito Alta & $\begin{array}{l}\text { Coeficiente } \\
\text { de Correlação }\end{array}$ & $\begin{array}{l}\text { Nível de } \\
\text { Significância }\end{array}$ \\
\hline Índice de Salubridade & 4,54 & 4,49 & 4,20 & 4,21 & $-0,22$ & $p<0,001$ \\
\hline Índice de Desenvolvimento Humano & 0,66 & 0,64 & 0,61 & 0,60 & $-0,27$ & $p<0,001$ \\
\hline Índice de Condições de Vida & 0,70 & 0,69 & 0,66 & 0,65 & $-0,28$ & $p<0,001$ \\
\hline Índice de Desenvolvimento Infantil & 0,58 & 0,57 & 0,54 & 0,52 & $-0,27$ & $p<0,001$ \\
\hline Renda Familiar Média per capita & 1,02 & 0,91 & 0,77 & 0,79 & $-0,25$ & $p<0,001$ \\
\hline Índice de Theil & 0,48 & 0,52 & 0,54 & 0,55 & 0,12 & $p=0,032$ \\
\hline Renda insuficiente & $47,9 \%$ & $51,8 \%$ & $59,0 \%$ & $60,1 \%$ & 0,31 & $p<0,001$ \\
\hline Aglomeração domiciliar & $25,7 \%$ & $25,8 \%$ & $28,9 \%$ & $31,2 \%$ & 0,19 & $p=0,001$ \\
\hline Domicílios construídos com materiais duráveis & $99,0 \%$ & $98,6 \%$ & $97,6 \%$ & $97,2 \%$ & $-0,17$ & $p=0,002$ \\
\hline Domicílios com ligação de água adequada & $87,7 \%$ & $88,0 \%$ & $85,0 \%$ & $83,2 \%$ & $-0,20$ & $p<0,001$ \\
\hline Coeficiente de analfabetismo & $18,0 \%$ & $18,9 \%$ & $20,3 \%$ & $21,5 \%$ & 0,15 & $p=0,007$ \\
\hline Anos de estudo & 4,05 & 3,79 & 3,44 & 3,31 & $-0,29$ & $p<0,001$ \\
\hline Crianças (7-14 anos) sem escola & $16,9 \%$ & $18,9 \%$ & $20,3 \%$ & $21,1 \%$ & 0,17 & $p=0,003$ \\
\hline Defasagem escolar média (em anos) & 1,62 & 1,62 & 1,73 & 1,76 & 0,16 & $p=0,005$ \\
\hline $\begin{array}{l}\text { Crianças ( } 7-14 \text { anos) com mais de } 1 \text { ano } \\
\text { de atraso escolar }\end{array}$ & $44,6 \%$ & $45,8 \%$ & $49,1 \%$ & $50,4 \%$ & 0,19 & $p=0,001$ \\
\hline Crianças (7-14 anos) que trabalham & $13,7 \%$ & $15,7 \%$ & $16,2 \%$ & $18,0 \%$ & 0,18 & $p=0,002$ \\
\hline
\end{tabular}

A relação entre o CPO-D e os diversos indicadores sociais utilizados neste estudo, pôde também ser evidenciada através dos mapas apresentados na Figura 1, nos quais são destacadas as áreas ocupadas pelos municípios com pior condição nos indicadores estudados, possibilitando a constatação visual de suas áreas de superposição.

Visando a obtenção de resultados suplementares não apresentados em elementos gráficos ou tabulares, foram avaliadas as médias de CPOD entre os municípios que apresentavam flúor no sistema de abastecimento público de água, em relação aos que não o apresentavam, encontrando-se diferença significante $(p=0,004)$. Os valores obtidos foram 5,05 (IC 95\%: 4,85-5,25) para os municípios que fluoravam a água e 5,95 $(5,32-6,58)$ para os que não o faziam. A análise de correlação do CPO-D com o porcentual de domicílios ligados à rede geral de abastecimento de água, indicou que, para o grupo de municípios sem água fluorada, não houve correlação significativa entre o CPO-D aos 12 anos e o porcentual de domicílios ligados à rede de águas 
alta e altíssima prevalência de cáries

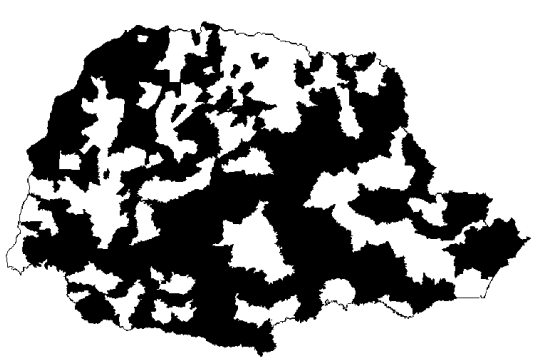

renda familiar per capita $<0,8$ s.m.

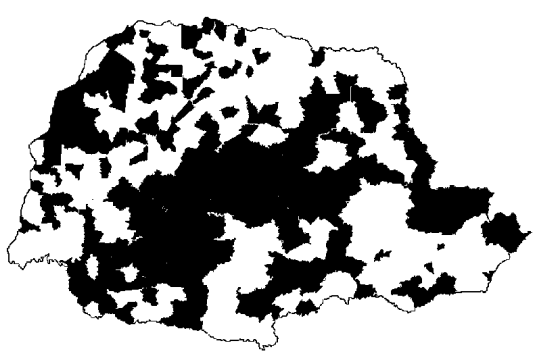

índice de salubridade $<4,35$

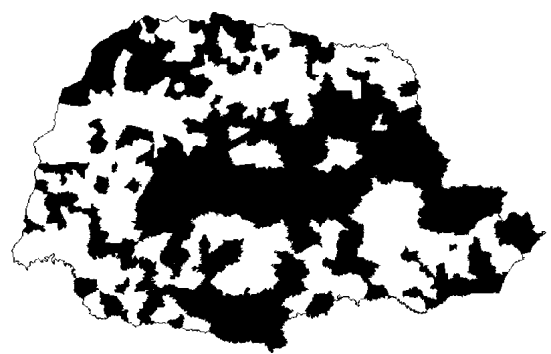

escolaridade média $<3,5$ anos

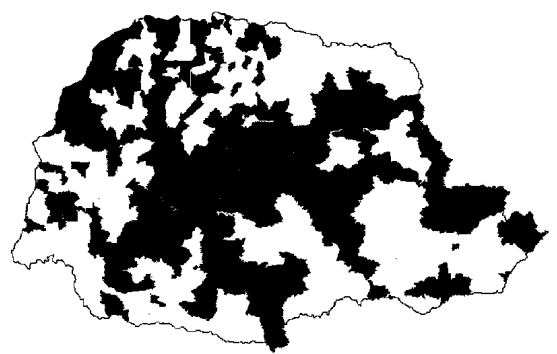

índice de desenvolvimento humano $<0,6$

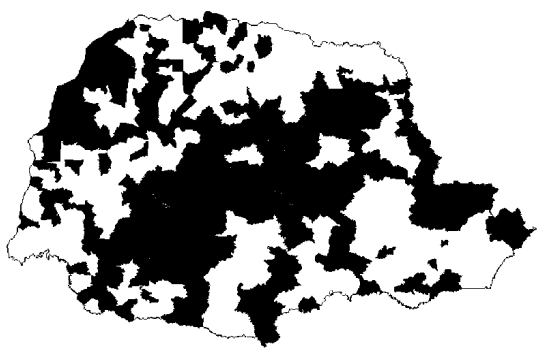

instalações adequadas de água $<87 \%$

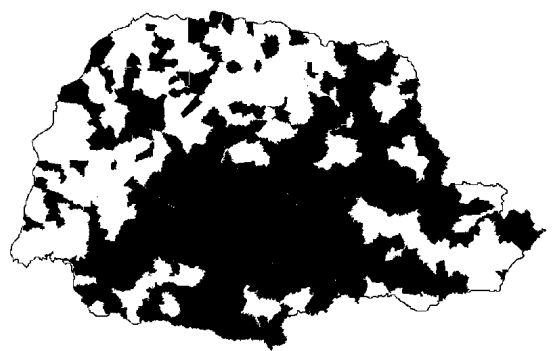

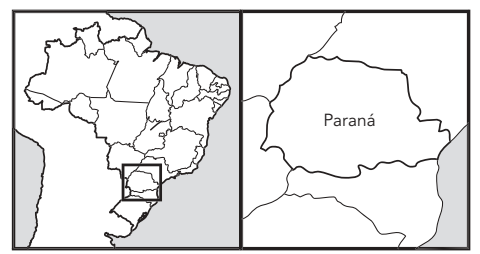

$(\mathrm{p}=0,15)$. Porém, para o segundo grupo, observou-se correlação negativa ( $R=-0,309, p<0,001)$, indicando que quanto maior o porcentual de domicílios ligados à rede de água, menores os valores do CPO-D nos municípios.

\section{Discussão}

Localizado na Região Sul do Brasil, o Estado do Paraná ocupa uma área de $199.323 \mathrm{~km} 2$. Em 1996, a população do Estado era de 9.003.804 habitantes, distribuídos em 371 municípios, com densidade demográfica de aproximadamente 45 habitantes/ km². Desses municípios, 48 haviam sido criados em 1996. Segundo o Censo demográfico de 1991 (IBGE, 1994), a po- pulação do Estado, residente nas áreas urbanas, representava $75,2 \%$ do total. Os indicadores econômicos do Paraná, revelam que o Produto Interno Bruto (PIB) per capita no ano de 1996, foi de 5.287 reais, ocupando o sexto lugar em importância econômica entre os estados brasileiros. A taxa de pobreza no Estado naquele ano foi de $20,01 \%$ e a taxa de desemprego de $5,67 \%$ (Brasil, 2001b). A taxa de alfabetização em 1996, foi de $88,28 \%$ (IBGE, 2001).

Na Tabela 1, observa-se que a média de CPO-D para o Estado foi de 5,15, condição considerada como de alta prevalência de cárie. Estes resultados contrastam com o valor de 2,23 atribuído ao Estado do Paraná no levantamento de 1996, que havia apenas levado em consideração a cidade de Curitiba. Cabe sublinhar o 
el evado contraste entre as duas observações produzidas para o Estado no mesmo período, passando a prevalência da cárie dentária no Paraná de baixa $(2,23)$ para alta $(5,15)$.

A marca da desigualdade é um fato que chama a atenção no cenário brasileiro. As diferenças estão presentes não apenas entre as grandes regiões do país, mas também no interior das diversas regiões de um Estado, e até mesmo nos diferentes bairros de um município. No caso do Paraná, nota-se que suas várias regiões possuem características históricas, sociais, econômicas e culturais distintas, o que faz com que sua população apresente perfis de saúde diferentes, prevalecendo piores indicadores para os grupos com pior qualidade de vida (Figura 1). Essa situação também foi identificada por Moysés (1997), ao analisar a distribuição da cárie dentária em Curitiba, capital do Estado. O autor apontou a heterogeneidade na distribuição do agravo em diferentes áreas do município, com médias inferiores de CPO-D nas regiões com melhor qualidade de vida, e piores índices de cárie em distritos mais submetidos à privação social, nos quais predominavam famílias migrantes, oriundas principalmente do interior agrícola do Paraná ou de pequenos municípios, carregando consigo uma herança epidemiológica fruto da desassistência e das baixas condições de vida.

Observação similar pode ser observada na Tabela 2, a qual registra que a maioria dos municípios de pequeno porte apresenta padrões de al ta e muito alta prevalência de cárie, mostrando ainda uma inversão nos padrões, em comparação com os municípios de grande porte. Situação semel hante foi constatada por dois estudos em que municípios do Estado de São Paulo foram analisados. O primeiro deles, referente ao período de 1990 a 1995 (Peres et al., 1997), identificou a maioria dos municípios de pequeno e médio porte como sendo de alta e muito alta prevalência de cárie, enquanto os de grande porte, concentraram-se majoritariamente nos níveis de moderada a al ta prevalência de cárie. O segundo estudo, refere-se ao levantamento epidemiológico realizado pela Secretaria de Estado da Saúde de São Paulo, em 1998, e também constatou que os valores de CPO-D tendem a ser menores nos municípios de maior porte demográfico. Porém, os municípios do Estado de São Paulo, apresentaram médias inferiores às dos municípios do Paraná: 4,15 para os municípios de pequeno porte; 4,02 para os de médio porte e 2,71 para os de grande porte (SES-SP, 1999).

A correlação entre renda familiar e CPO-D médio aos 12 anos, destaca o impacto dessa va- riável: cidades com renda média mais elevada tendem a apresentar menores médias de CPOD, uma observação também efetuada por Loretto et al . (2000). Nadanovsky (2000) ponderou que pessoas com baixa renda familiar tendem a ingerir mais açúcar. O mesmo concluiu Furlani (1993), em Jaraguá do Sul, Estado de Santa Catarina, ao demonstrar que as famílias de mais baixa renda apresentavam o hábito de ingerir melado em grande quantidade e freqüência. As mães de classes sociais de menor renda, tendem a amamentar seus filhos por menos tempo, além de acrescentar açúcar e farinhas às mamadeiras. Há que se considerar, quanto a aspectos dietético-nutricionais, que fatores culturais contribuem de modo importante para a manutenção de hábitos al imentares deletérios.

Além da renda familiar, a inserção social tem influência direta na prevalência da cárie dentária (Furlani, 1993; Nadanovsky, 2000; Peres et al., 2000). Indivíduos com grau de escolaridade maior, tendem a escovar seus dentes mais vezes, e suas crianças começam a ter seus dentes higienizados mais precocemente, com impacto significativo na saúde bucal. Além disso, a disponibilidade de instalações sanitárias adequadas, favorece às práticas de higiene pessoal .

Observou-se, neste estudo, correlação negativa entre o CPO-D e os porcentuais de domicílios ligados a rede de água. Este achado pode ser interpretado sob dois ângulos: tanto pode expressar níveis de cáries menos elevados em condições de melhor desenvolvimento social, quanto pode ser um indício de êxito da estratégia de adição de flúor às águas de abastecimento público (U.S. Department of Health and Human Services, 1999). Cabe assinalar, que dados da SES-PR mostram que $85,7 \%$ dos municípios apresentavam fluoretação das águas de abastecimento público em 1996 (SES-PR, 2001). O excesso bruto de 0,9 observado para o CPO-D aos 12 anos nos municípios não fluoretados, corresponde a, em média, 900 dentes a mais com ataque de cáries para cada 1.000 crianças da idade considerada, indicação consistente com a avaliação positiva da efetividade econômica da medida, conforme apontada por Griffin et al. (2001).

Como indicação adicional, observou-se que, para os municípios com flúor, o esforço de aumentar em $10 \%$ a proporção de domicílios ligados à rede de água, implicaria uma redução bruta de 0,274 (IC 95\%: 0,264-0,284) no CPO-D aos 12 anos. Em outras palavras, para cada 1.000 crianças de 12 anos nesses municípios, em média 274 dentes deixariam de sofrer ataque de cáries, com redução concomitante em sofri- 
mento evitável, prejuízo à qualidade de vida e demanda por serviços de restauração dentária.

Tendo em vista que as principais marcas comerciais de dentifrícios adicionaram fluoretos em sua composição desde 1988, corroborase a efetividade do acesso à água fluorada como diferencial para a prevenção da cárie dentária. Resultados semelhantes foram relatados por outros autores (Furlani, 1993; Jones \& Worthington, 2000), os quais atribuem à essa medida, a capacidade de reduzir sensivelmente o impacto negativo das desigualdades sócio-econômicas sobre a prevalência de cárie dentária, ainda que, não obstante, não as possam neutralizar.

\section{Referências}

ANDRADE, M., 2000. Como está o sorriso do Brasil? Revista da ABO Nacional, 8:134-135.

DATASUS (Departamento de Informática do SUS), 2000. Tabulador de Informações de Saúde para AmbienteWindows, Tabwin, Versão 1.4. Brasília: Ministério da Saúde.

DATASUS (Departamento de Informática do SUS), 2001. Informações deSaúde, 26 março 2001 \tttp:// www.datasus.gov.br/ >

FURLANI, P. A., 1993. Influências dos Aspectos Sociais eCulturais na Prevalência da Cárie Dentária em Escolares de Jaraguá do Sul. Curitiba: Universidade Federal do Paraná.

GRIFFIN, S. O.; JONES, K. \& TOM AR, S. L., 2001. An economic evaluation of community water fluoridation. Journal of Public Health Dentistry, 61:7886.

IBGE (Fundação Instituto Brasileiro de Geografia e Estatística), 1994. Censo Demográfico, Brasil, 1991. Rio de Janeiro: IBGE.
IBGE (Fundação Instituto Brasileiro de Geografia e Estatística), 2001. Sistema IBGE de Recuperação Automática - SIDRA, 26 março 2001 «ttp:// www.sidra.ibge.gov.br/>.

JONES, C. M. \& WORTHINGTON, H., 2000. Water fluoridation, poverty and tooth decay in 12-year-old children. Journal of Dentistry, 28:389-393.

JOHNSON, R. A. \& WICHERN, D. W., 1998. Applied Multivariate Statistical Analysis. Upper Saddle River: Prentice Hall.

LORETTO, N. R. M.; SEIXAS, Z. A.; JARDIM, M. C. \& BRITO, R. L., 2000. Cárie dentária no Brasil: Alguns aspectos sociais, políticos e econômicos. Revista da ABO Nacional, 8:45-49.

MARCENES, W. \& BONECKER, M. J. S., 2000. Aspectos epidemiológicos e sociais das doenças bucais. In: Promoção de Saúde Bucal na Clínica Odontológica (Y. P. Buischi, org.), pp. 75-98, São Paulo: Artes Médicas.

MARTINS, M. D.; ARAÚJO, R. G. D. \&VELOSO, N. F., 
1999. Avaliação das necessidades de tratamento odontológico de crianças de baixa renda. Jornal Brasileiro de Odontopediatria e Odontologia do Bebê, 2:132-136.

MINAYO, M. C. S.; HARTZ, Z. M. A. \& BUSS, P. M., 2000. Qualidade de vida e saúde: Um debate necessário. Ciência \& Saúde Coletiva, 5:7-18.

MOYSÉS, S., 1997. O conceito de promoção de saúde na construção de sistemas de atenção em saúde bucal coletiva. In: Promoção deSaúdeBucal (L. Kriger, org.), pp. 371-407, São Paulo: Artes Médicas.

MS (Ministério da Saúde),1988. Levantamento Epidemiológico em Saúde Bucal: Brasil, Zona Urbana, 1986. Brasília: Divisão Nacional de Saúde Bucal, Centro de Documentação, Ministério da Saúde.

MS (Ministério da Saúde), 2001. Área Técnica de Saúde Bucal. Levantamento Epidemiológico em Saúde Bucal: Cárie Dental, Capitais, 1996, 26 março 2001 ४tttp:// www.saude.gov.br/ >

MURRAY, J. J., 1992. O Uso Correto de Fluoretos na Saúde Pública. São Paulo: Organização Mundial da Saúde/ Editora Santos.

NADANOVSKY, P., 2000. O declínio da cárie. In: Saúde Bucal Coletiva (V. G. Pinto, org.), pp. 341-351, São Paulo: Editora Santos.

NARVAI, P. C.; CASTELLANOS, R. A. \& FRAZÃO, P., 2000. Prevalência de cárie em dentes permanentes de escolares do Município de São Paulo, SP, 1970-1996. Revista de Saúde Pública, 34:196-200.

NARVAI, P. C., FRAZÃO, P. \& CASTELLANOS, R. A., 1999. Declínio na experiência de cárie em dentes permanentes de escolares brasileiros no final do século XX. Odontologia e Sociedade, 1:25-29.

OMS (Organização Mundial da Saúde), 1999. Levantamentos Básicos em SaúdeBucal. 4ạ Ed. São Paulo: Editora Santos.

PERES, K. G. A.; BASTOS, J. R. M. \& LATORRE, M. R. D. O., 2000. Severidade de cárie em crianças e relação com aspectos sociais e comportamentais. Revista de Saúde Pública, 34:402-408.

PERES, M. A. A.; NARVAI, P. C. \& CALVO, M. C. M., 1997. Prevalência de cárie dentária em crianças aos doze anos de idade, em localidades do Estado de São Paulo, Brasil, período 1990-1995. Revista deSaúdePública, 31:594-600.

PINTO, V. G., 1996. Índice de cárie no Brasil e no mundo. Revista Gaúcha de Odontologia, 44:8-12.

PINTO, V. G., 1997. Epidemiologia das doenças bucais no Brasil. In: Promoção de Saúde Bucal (L. Kriger, org.), pp. 24-41, São Paulo: Artes Médicas.
PINTO, V. G., 2000. Saúde Bucal Coletiva. 4ạ Ed. São Paulo: Santos.

PNUD (Programa das Nações Unidas para o Desenvolvimento)/INSTITUTO DE PESQUISAS DE ALIMENTAÇÃO E NUTRIÇÃO/FJP (Fundação João Pinheiro)/IBGE (Fundação Instituto Brasileiro de Geografia e Estatística), 1997. Atlas do Desenvolvimento Humano no Brasil. Brasília: PNUD.

SES-PR (Secretaria de Estado da Saúde do Paraná), 1996a. Cárie Dentária - 12 anos: Perfil Epidemiológico no Estado do Paraná. Curitiba: SES-PR.

SES-PR (Secretaria de Estado da Saúde do Paraná), 1996b. Levantamento Epidemiológico da Cárie Dentária em Escolares de 12 anos: Manual delnstruções. Curitiba: SES-PR.

SES-PR (Secretaria de Estado da Saúde do Paraná), 2001. Dados sobre Fluoração das Águas de Abastecimento Público no Estado do Paraná. Curitiba: SES-PR.

SES-SP (Secretaria de Estado da Saúde de São Paulo), 1999. Levantamento Epidemiológico em Saúde Bucal: Estado de São Paulo, 1998. São Paulo: SESSP/Universidade de São Paulo.

SPSS INCORPORATION, 1997. SPSS for Windows. Statistical Packagefor the Social Sciences. Release 8.0. Chicago: SPSS Inc.

STROZZI, J. B., 1997. SUS Pense: Uma Reflexão Epidemi ológica sobre o SUS e Outras Aplicações Acadêmicas. Londrina: Centro Brasileiro de Estudos de Saúde.

UNICEF (Fundo das Nações Unidas para a Infância), 2001. Situação da Infância Brasileira 2001. Brasília: UNICEF.

U. S. DEPARTMENT OF HEALTH AND HUM AN SERVICES, 1999. Achievements in Public Health, 1900-1999: Fluoridation of drinking water to prevent dental caries. MMWR, 48:933-940.

VAUGHAN, J. P. \& MORROW, R. H., 1997. Epidemiologia para os Municípios. 2a Ed. São Paulo: Editora Hucitec.

WEYNE, S. C., 1997. A construção do paradigma de promoção de saúde: Um desafio para as novas gerações. In: Promoção de Saúde Bucal (L. Kriger, org.), pp. 1-26, São Paulo: Artes Médicas.

Recebido em 14 de maio de 2001

Versão final reapresentada em 16 de outubro de 2001

Aprovado em 20 de dezembro de 2001 